\title{
Research on Balanceable Path planning Method for Mobile Wireless Sensor Network
}

\author{
Zhenya Zhang $^{1, a}$, Xuewen Yuan ${ }^{1, b}$, Weili Wang ${ }^{1, b}$, Hongmei Cheng ${ }^{2, d}$ \\ ${ }^{1}$ Anhui provincial Key Laboratory of Intelligent Building, Anhui Jianzhu University, Hefei, 230022, \\ China \\ ${ }^{2}$ School of Management, Anhui Jianzhu University, Hefei 230022, China \\ aemail:zyzhang@ahjzu.edu.cn, ${ }^{b}$ email:853870932@qq.com cemail:ustc301@sina.com, demail:ustc \\ 017@sina.com
}

Keywords: Sweep Coverage; Balance Factor; Travel Salesman Problem; Evolutionary Computation Algorithms

\begin{abstract}
Path planning problem of low cost effective sweep coverage for mobile wireless sensor network (MWSN) is one MTSP problem and the number of mobile nodes is the number of travel sales man in the MTSP problem. To keep the balance of moving path for each mobile node in sweep coverage of MWSN, features of mobile node's burden are discussed and balance factor are presented to measure the balance of load for sweep coverage in this paper. Fast balance factor is proposed for evolutionary computation algorithms based path planning. Experimental results show that the balance of moving path planed by our proposed method is better.
\end{abstract}

\section{Introduction}

In sweep coverage [1-3] of mobile wireless sensor network (MWSN) [1], each mobile node moves along one point of interesting sequence as its moving path [1][2]. In sweep coverage of MWSN, moving path for each mobile node should be planed rationally. The path plan problem for mobile nodes in low cost sweep coverage is one multiple travel salesman problem (MTSP)[1] with the maximum visiting deadline time $T$ as constrains[3][4].

To measure the difference of mobile node on cost, balance factor is defined in this paper. With balance factor, fitness function of evolutionary computation algorithms such as genetic algorithm [5] can be designed when the path planning problem of sweep coverage is solved by evolutionary computation algorithms. In the following, balance factor for path planning of sweep coverage is discussed at section2. And fitness function based on balance factor for evolutionary computation algorithms is given at section3. Experimental results are shown at section4. Conclusion and future work are given at section5.

\section{Loads in Sweep Coverage}

Let $P=\left\{P_{i} \in \Omega \subseteq R^{2}, i=1,2 \cdots n\right\}$ be set for point of interesting (POI) and $S=\left\{s_{i}, \mathrm{i}=1,2 \ldots \mathrm{m}\right\}$ be the set of all mobile nodes in MWSN. And let $P P_{\mathrm{j}}=<P_{\mathrm{j} 1}, P_{\mathrm{j} 2} \ldots P_{\mathrm{js}}>$ be the moving path of mobile node $s_{j} \in S$ in one low cost effective sweep coverage. Because the sweep coverage is effective, $P=\bigcup_{j=1}^{m} P P_{j}, P P_{i} \cap P P_{j}=\varnothing$, and $P_{\mathrm{j} 1}=P_{\mathrm{js}}$ where $1 \leq \mathrm{j} \leq \mathrm{m}$. And because the sweep coverage is also low cost, the moving path for each mobile node in sweep coverage of MWN is one Hamilton loop.

In MWSN, there are 3 kind actions of mobile nodes for their behavior. The first is moving action which is the moving behavior of one mobile node from one POI to another adjacent POI. The second is information sampling action which is the information sampling behavior of one mobile node near one POI. The third is other auxiliary action which is some other behaviors of one mobile node. Because there are some loads for the happening of each kind action, some cost should be paid for the maintenance of sweep coverage by MWSN. Because each kind of action of one mobile node 
is happening during the sweep process which the mobile node is moving along its moving path in sweep coverage, loads of one mobile node is related to its moving path. Let $P P_{\mathrm{j}}=<P_{\mathrm{j} 1}, P_{\mathrm{j} 2} \ldots P_{\mathrm{js}}>$ be the moving path of mobile node $s_{\mathrm{j}}$ in one low cost effective sweep coverage and let $m L o a d(\mathrm{i}, \mathrm{j}) \geq 0$ be the moving action load of one mobile node when the mobile node moves from POI $P_{\mathrm{i}}$ to POI $P_{\mathrm{j}}$, sLoad(i) $\geq 0$ be the information sampling load when the mobile node samples data near one POI, oLoad (i) $\geq 0$ be the loads for other auxiliary action of one mobile node. Let $L m, L s$ and $L o$ be the summation of loads for moving action, information sampling action and other auxiliary action of one mobile node when the mobile node moves along its moving path. If Load is the summation of all load of mobile node $s_{\mathrm{i}}$ along moving path $P P_{\mathrm{j}}$, some equations can be defined as following.

$$
\begin{aligned}
& \operatorname{Lm}\left(P P_{j}\right)=\sum_{i=j_{1}}^{j_{s}-1} m \operatorname{Load}(i, i+1) \\
& \operatorname{Ls}\left(P P_{j}\right)=\sum_{i=j_{1}}^{j_{s}-1} s L o a d(i) \\
& \operatorname{Lo}\left(P P_{j}\right)=\sum_{i=j_{1}}^{j_{s}-1} o L o s t(i) \\
& \operatorname{Load}\left(s_{i}, P P_{j}\right)=\left(\begin{array}{lll}
c_{1}\left(s_{i}\right) & c_{2}\left(s_{i}\right) & c_{3}\left(s_{i}\right)
\end{array}\right)\left(\begin{array}{c}
\operatorname{Lm}\left(P P_{j}\right) \\
L s\left(P P_{j}\right) \\
L O\left(P P_{j}\right)
\end{array}\right) \\
& \operatorname{Load}\left(s_{i}, P P_{j}\right)=\left(\begin{array}{lll}
c_{1} & c_{2} & c_{3}
\end{array}\right)\left(\begin{array}{c}
\operatorname{Lm}\left(P P_{j}\right) \\
\operatorname{Ls}\left(P P_{j}\right) \\
\operatorname{Lo}\left(P P_{j}\right)
\end{array}\right) \\
& B^{*}(L)=\frac{\max (L)-\min (L)}{\max (L)}
\end{aligned}
$$

In equation (4), $c_{1}\left(s_{\mathrm{i}}\right), c_{2}\left(s_{\mathrm{i}}\right)$ and $c_{3}\left(s_{\mathrm{i}}\right)$ are coefficients for moving action load, information sampling action load and other auxiliary action of mobile node $s_{\mathrm{i}}$. In equation(4), $c_{1}\left(s_{\mathrm{i}}\right) \geq 0, c_{2}\left(s_{\mathrm{i}}\right) \geq 0$ and $c_{3}\left(s_{\mathrm{i}}\right) \geq 0$.

In sweep coverage of MWSN, if more POIs are in moving path for one mobile node, load of the mobile node is greater. Furthermore, if the length of moving path for one mobile node is longer, the load of the mobile node is greater.

If structure, function and life-span of each mobile node in a MWSN are homothetic, each load coefficients is independent of mobile node. With this assumption, $c_{1}\left(s_{\mathrm{i}}\right), c_{2}\left(s_{\mathrm{i}}\right)$ and $c_{3}\left(s_{\mathrm{i}}\right)$ in equation(4) can be denoted as $c_{1}, c_{2}$ and $c_{3}$ and equation(4) can be simplified as equation(5). In equation(5), $c_{1} \geq 0, c_{2} \geq 0, c_{3} \geq 0$.

Because $L m$ is caused by mechanical motion meanwhile $L s$ and $L o$ are mainly caused by electronic operation, $\mathrm{Lm}$ is the main parts of load of one mobile node in sweep coverage. If any load caused by electronic operation is ignored, only $\mathrm{Lm}$ need to be considered when the load of one mobile node is calculated according to equation (4) or equation (5).

In sweep coverage of MWSN, effective sweep coverage means that each POI is visited by at least one mobile node along its moving path before stop time. Let $P P_{\mathrm{i}}$ be the moving path of mobile node $s_{\mathrm{i}}$ and $L_{\mathrm{i}}$ be the abbreviation of $\operatorname{Load}\left(s_{\mathrm{i}}, P P_{\mathrm{i}}\right)$. If $\boldsymbol{L}=\left\{L_{\mathrm{i}} \mid \mathrm{i}=1,2 \ldots \mathrm{m}\right\}$ is the load set for mobile node set $\mathbf{S}=\left\{s_{\mathrm{i}} \mid \mathrm{i}=1,2 \ldots \mathrm{m}\right\}$ in sweep coverage, $E(L)=\frac{1}{m} \sum_{j=1}^{m} L_{j}$ is the mean load of mobile node in sweep coverage and $D(L)=\frac{1}{m} \sum_{i=1}^{m}\left(L_{i}-E(L)\right)^{2}$ is the variance for mobile node's load. $D(L)$ can be used to measure whether load of each mobile node is balanceable.

Definition1: Let $S=\left\{s_{i}, \mathrm{i}=1,2 \ldots \mathrm{m}\right\}$ be the mobile node set for sweep coverage of MWSN and 
$\boldsymbol{L}=\left\{L_{\mathrm{i}} \mid \mathrm{i}=1,2 \ldots \mathrm{m}\right\}$ be the load set for each mobile node. If $d_{\mathrm{ij}}=\left|L_{\mathrm{i}}-L_{\mathrm{j}}\right|$ where $\mathrm{i}, \mathrm{j}=1,2 \ldots \mathrm{m}$, $B(L)=\frac{1}{m^{2}} \sum_{i=1}^{m} \sum_{j=1}^{m} d_{i j}$ is the balance factor for sweep coverage of MWSN.

For balance factor defined at definition1, three conclusions as following can be proved. Firstly, $B(L) \geq 0$. Secondly, $B(L)=0$ if the load of each mobile node is same. Thirdly, if difference for load of each mobile node is greater/less, $\mathrm{B}(L)$ is greater/less. Same conclusions can be proved for $B^{*}(L)$ defined at equation(6). It is clearly that load of mobile node in sweep coverage of MWSN is more balanceable if the value of $B(L)$ or $B^{*}(L)$ is more less.

If there are $m$ mobile nodes in sweep coverage of MWSN, the time complexity of $\mathrm{B}(L)$ computing should be $\mathrm{O}\left(\mathrm{m}^{2}\right)$ according to equation(4). On the other hand, because $B^{*}(L)$ defined by equation(6) has same characteristics with $\mathrm{B}(L)$ on the measuring of balance for sweep coverage, $B^{*}(L)$ can be treated as one kind of balance factor for sweep coverage. And because the time complexity of $B^{*}(L)$ is $\mathrm{O}(m)$ when there are $m$ mobile nodes in sweep coverage, $B^{*}(L)$ is the fast balance factor for sweep coverage of MWSN. It is obvious that $B^{*}(L) \in\left[\begin{array}{ll}0 & 1\end{array}\right]$. And more less the value of $B^{*}(L)$ is, more balanceable of the sweep coverage on mobile node's loads is.

\section{Balance Factor Based Fitness Function for Evolutionary Computation Algorithms}

In fact, the balance status of mobile node's load in sweep coverage of MWSN can be measured by $D(L)$ which is the variance of load sequence $L=\left\{L_{\mathrm{i}}, \mathrm{i}=1,2 \ldots \mathrm{m}\right\}$ for all $m$ mobile nodes in sweep coverage. Because $\mathrm{D}(L)$ is equivalent to balance factor $\mathrm{B}(L)$ which is defined at definition $1, \mathrm{~B}(L)$ is the quantitative indicator for balance status of mobile node's load in sweep coverage of MWSN. Because $B^{*}(L)$ defined by equation(6) has same characteristic with $\mathrm{B}(L)$ on the measuring of balance status of sweep coverage, $B^{*}(L)$ can be used to measure the balance status of sweep coverage. And because the time complexity of $B^{*}(L)$ is $\mathrm{O}(\mathrm{m})$ which is less either than time complexity of $B(L)$ or than time complexity of $D(L)$, balance status of sweep coverage measured by $B^{*}(L)$ can be computed quickly.

Algorithm1: $B^{*}(L)$ based fitness function of evolutionary computation algorithms for the solving of multiple travel salesman problem

Input: pathCode, moving path sequence for all mobile nodes; distance matrix $\boldsymbol{D}=\left(d_{\mathrm{ij}}\right)_{\mathrm{n} \times \mathrm{n}}$ where $d_{\mathrm{ij}}$ is the distance from $\mathrm{P}_{\mathrm{i}}$ to $\mathrm{P}_{\mathrm{j}}$; $m$, the number of travel salesman

Output: $f$, the fitness value based on pathCode

1) Part sequence pathCode into $m$ segments with pathCode[n+1], pathCode[n+2] ... pathCode $[n+m-1]$ as segmentation points. Each segment is denoted as $P P_{\mathrm{i}}$ which is one POI sequence, $\mathrm{i}=1,2 \ldots \mathrm{m}$.

2) $\mathrm{L}=\Phi$;

3) for $\mathrm{i}=1$ to $\mathrm{m}$ step by 1

4) calculate $L_{\mathrm{i}}$, the load of $i$ th mobile node according to $\boldsymbol{D}$ and equation(5).

5) $\quad L=L \bigcup L_{i}$

6) end for

7) $f=\frac{\max (L)-\min (L)}{\max (L)}$

In algorithm1, pathCode is treated as one array with $n+m-1$ element where $m$ is the number of travel salesman and $n$ is the number of POI. And in MTSP based path planning for sweep coverage of MWSN, the number of travel salesman is the number of mobile nodes in sweep coverage of MWSN. Element in each pathCode[i], $\mathrm{i}=1,2 \ldots \mathrm{n}$, is the ID of one POI and pathCode[1...n] is one permutations of all $n$ POIs. Because pathCode is the record for the moving path of each travel salesman, pathCode[1...n] should be parted into $\mathrm{m}$ segments with pathCode[n+1], pathCode[n+2] ...pathCode[n+m-1] as segmentation points and $P P_{\mathrm{i}}$, the $i$ th segment is the travel POI sequence for the ith travel salesman, $i=1,2 \ldots m$. 


\section{Experimental Results and Analysis}

In our experiment, moving path among some POIs in 10x10 area for each mobile nodes in sweep coverage of MWSN is planned. Genetic algorithm is used to solve MTSP problem and the fitness function for genetic algorithm is implemented according to algorithm1. In the implementation of genetic algorithm, the number of population is 80 ; the crossover probability is 0.01 and the mutation probability 0.001 .

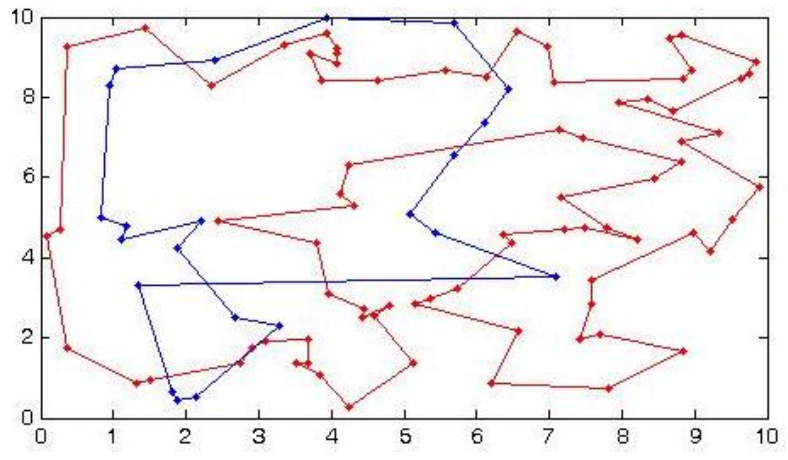

Fig1: the first case $\left(\mathrm{B}^{*}=0.3270 \quad \mathrm{~B}=27.7401\right)$

The balance status of path planning for sweep coverage with 2 mobile nodes among 100 POIs are shown at fig1. In fig1, B is balance factor and $\mathrm{B}^{*}$ is fast balance factor. It is obvious that the balance of moving path with fast balance factor is good.

\section{Conclusion}

The longevity of mobile nodes is depended on load of each mobile node and the longevity of mobile wireless sensor network is depended on whether loads of all mobile nodes are balanceable. In this paper, balance factor is introduced to plan moving path for all mobile nodes with the balance of loads as constrains. To estimate the balance status of moving path quickly, fast balance factor is defined in this paper too. With fast balance factor, balanceable moving path of each mobile node can planned with evolutionary computation algorithms quickly.

\section{Acknowledgements}

This work is partially supported by the National Natural Science Foundation of China (No.61340030, 61300060), Special Foundation for Young Scientists of Anhui Province (2013SQRL043ZD) and Science and technology project of Ministry Housing and Urban-Rural Development Department in (2014-K8-061).

\section{Reference}

[1] Mo Li, Cheng, Weifang Cheng, Kebin Liu, Yunhao Liu, Sweep coverage with mobile sensors[J], IEEE Transactions on Mobile Computing, v2011.11, v10n11:1534-1545

[2] Zhenya Zhang, Jia Liu, Hongmei Cheng, Qiansheng Fang, Research on the Minimum Number of Mobile Node for Sweep Coverage in Wireless Sensor Network based on Genetic Algorithm[J], Journal of Chinese Computer Systems, 2013.10,v34n10:2388-2392

[3] Zhenya Zhang,Yan Chen, MTSP based solution for minimum mobile node number problem in sweep converge of wireless sensor network, International Conference on Computer Science and Network Technology,2011,vl:1827-1830

[4] Yan Cheng, Research on methods for getting the minimum number of mobile sensors in WSN sweep coverage, Anhui University of Architecture, 2012

[5] Alok Singh, Anurag Singh Baghel, A new grouping genetic algorithm approach to the multiple traveling salesperson problem, Soft Computing, 2009, v13n1:95-101 